\title{
Anesthetic Management of a Child with Hyper-IgE Syndrome for Pneumatocele Excision: A Case Report
}

\author{
Deepali P Thakur ${ }^{1}$, Anila D Malde ${ }^{2}$
}

\begin{abstract}
We describe a child of hyperimmunoglobulin-E syndrome (HIES) with bilateral pneumatoceles for an elective right thoracotomy for pneumatocele excision. HIES is a rare primary immunodeficiency characterized by the triad of markedly elevated serum levels of immunoglobulin $E(>2000 \mathrm{IU} / \mathrm{mL}$ ) with peripheral eosinophilia, recurrent staphylococcal infections of the skin and lungs, and chronic eczema. A 10-year-old male child was presented with recurrent skin abscesses and lung infections, leading to extensive bilateral pneumatocele formation. Such children present unique anesthetic challenges in terms of immunodeficiency, multiple hospital admissions, difficult intravenous (IV) access, and poor pulmonary compliance affecting intraoperative ventilation. Along with pediatric lung isolation techniques, thorough asepsis, good perioperative analgesia and high index of suspicion for pneumothorax are essential components of successful anesthetic management.

Keywords: Anesthesia, Hyperimmunoglobulin-E syndrome, Pneumatocele, Thoracotomy.

Research and Innovation in Anesthesia (2019): 10.5005/jp-journals-10049-0059
\end{abstract}

\section{INTRODUCTION}

Hyperimmunoglobulin-E syndrome (HIES, Job's syndrome) is a rare primary immunodeficiency first described by Davis et al. ${ }^{1}$ It is a multisystem disorder caused by genetic mutations. ${ }^{2-4}$ The incidence of HIES is $<1: 10,00,000$ population. ${ }^{5,6}$ It presents as persistent cutaneous abscesses, recurring pneumonias, pneumatoceles, hypereosinophilia, craniofacial and bone growth changes, and elevated serum IgE levels. ${ }^{7-9}$ The objective of this report is to present a case of a rare multisystem disease and its anesthetic management in a patient undergoing right thoracotomy for a recurrent pneumatocele excision.

\section{Case Description}

A 10-year-old male child diagnosed with HIES, (weight: 20 kg; height: $120 \mathrm{~cm}$ ) with recurrent bilateral pneumatoceles was presented for an elective right thoracotomy for pneumatocele excision. The child was presented with high-grade fever and chronic productive cough. He had nine prior hospitalizations for infections, involving the skin, lung, and liver. Since birth, multiple times the drainage of cold abscesses from the neck, thigh, and face was done under antibiotic cover. He had a past history of empyema (three times) requiring chest drains. At 4 years of age, he was diagnosed to have HIES (IgE levels of $>50,800 \mathrm{IU} / \mathrm{mL}$, normal: 3-423 IU/mL). IgA, IgM, and IgG levels were normal. Following recurrent staphalococcal lung infections, he developed bilateral pneumatoceles. At nine years of age, he developed right sided spontaneous pneumothorax requiring emergency decompression with chest tube, liver abscess, and infective endocarditis. He had a prior history of right lower lobe pneumatocele excision under general anesthesia 4 months back with delayed extubation after 4 hours owing to a poor chest condition.

Physical examination revealed coarse facial features with flat nasal bridge, persistence of primary dentition, healed scars of chronic eczema, and grade II clubbing. He had difficult peripheral venous access. Child was normotensive with a pulse rate of $84 /$ minute, respiratory rate of $20 /$ minute, breath holding time of
1,2Department of Anaesthesiology, Lokmanya Tilak Municipal General Hospital and Medical College, Sion, Mumbai, Maharashtra, India

Corresponding Author:Anila D Malde,Department of Anaesthesiology, Lokmanya Tilak Municipal General Hospital and Medical College, Sion, Mumbai, Maharashtra, India, Phone: +91 9821085730, e-mail: dradmalde@yahoo.com

How to cite this article:Thakur DP, Malde AD. Anesthetic Management of a Child with Hyper-IgE Syndrome for Pneumatocele Excision: A Case Report. Res Inno in Anesth 2019;4(1):13-15.

Source of support: Nil

Conflict of interest: None

15 seconds, $\mathrm{SpO}_{2}$ on room air $95 \%$ and systolic murmur in the tricuspid area without clinical evidence of pulmonary hypertension. He had decreased breath sounds in bilateral bases, and rhonchi with coarse crepitations (right $>$ left side).

Investigations showed $\mathrm{Hb} 16.6 \mathrm{~g} / \mathrm{dL}$, white cell count $4700 / \mathrm{mm}^{3}$, high absolute eosinophil count $2920 / \mathrm{mm}^{3}$, normal platelets, normal liver, and renal function tests. Chest X-ray showed bilateral multiple pneumatoceles. High-resolution contrast CT chest demonstrated a large pneumatocele $(8.9 \times 7.4 \mathrm{~cm} \times 10.6)$ involving right middle and lower lobes extending along the anterior end of 2 nd to 6 th rib. An air-filled bulla $(5.2 \times 6.2 \times 5.1 \mathrm{~cm})$ involved posterior and lateral basal segments of the right lower lobe. The left lung parenchyma showed multiple thick-walled air-filled cavities measuring $3.7 \times$ $2.8 \times 2.4 \mathrm{~cm}$ (Figs 1 and 2). Fibronodular changes were also seen.

Pulmonary function test (PFT) revealed moderate restriction with small airway obstruction with forced vital capacity (FVC) $65 \%$ predicted, forced expiratory volume in 1 second (FEV1) $62 \%$ predicted, FEV1/FVC ratio $99 \%$ predicted, and peak expiratory flow rate (PEFR) $61 \%$ of predicted. Recent echocardiogram showed severe tricuspid regurgitation without pulmonary hypertension.

The child was on regular nebulization with ipratropium, salbutamol, and sulfamethoxazole-trimethoprim prophylaxis for optimization of the chest condition. Preoperatively, patient's respiratory functions were optimized as far as possible. However, due to the presence of bilateral pneumatoceles occupying more 
than $50 \%$ of lung parenchyma, a further improvement in his respiratory parameters was unlikely.

Written informed consent (ASA III physical status) was obtained from parents on explaining the risk of intraoperative pneumothorax and postoperative ventilatory support. 6 hours of starvation to solids was confirmed. Infective endocarditis prophylaxis (ceftriaxone $50 \mathrm{mg} / \mathrm{kg}$ IV) was given. In the operation room, cardioscope, noninvasive blood pressure (NIBP), and pulse oximeter $\left(\mathrm{SpO}_{2}\right)$ were applied. Before induction of anesthesia, a surgical trolley with chest tube was kept ready, in order to manage an inadvertent pneumothorax. Injection of glycopyrolate $(0.004 \mathrm{mg} / \mathrm{kg})$, midazolam $(0.05 \mathrm{mg} / \mathrm{kg})$ and fentanyl $(2 \mu \mathrm{g} / \mathrm{kg})$ was given IV after monitoring baseline parameters.

Anesthesia was induced with propofol $(50 \mathrm{mg})$ and sevoflurane in oxygen:air (50:50). The airway was secured with 26 Fr left-sided double lumen tube (DLT) after adequate muscle relaxation with vecuronium ( $2 \mathrm{mg}$ ). Lung isolation was confirmed by auscultation after alternate clamping of tracheal and brochial lumens. Left radial arterial and right internal jugular venous canulation was done for invasive monitoring and fluid management. Muscle relaxation was maintained with vecuronium infusion $(0.06 \mathrm{mg} / \mathrm{kg} / \mathrm{hour})$. Careful positioning of child was done perioperatively, with padding all pressure points. Anesthesia was maintained with oxygen-air $\left(\mathrm{FiO}_{2}\right.$ of 0.5 ) and sevoflurane (MAC 1-1.3) with volume-controlled ventilation (tidal volume 6-7 mL/kg, respiratory rate $30 /$ minute). Double lung ventilation with intermittent single lung ventilation of dependant lung as and when required was done while maintaining peak airway pressures below $18-20 \mathrm{~cm}$ of $\mathrm{H}_{2} \mathrm{O}$. A stethoscope was placed under
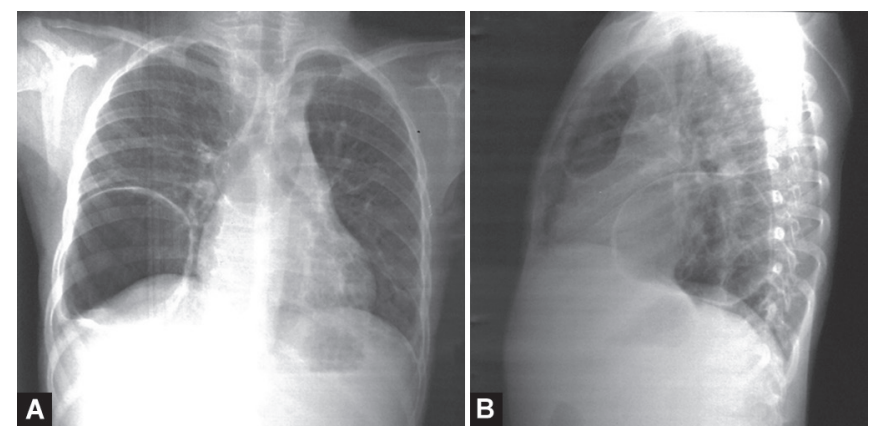

Figs $1 \mathrm{~A}$ and B: Chest X-ray: (A) PA view; (B) Right lateral view
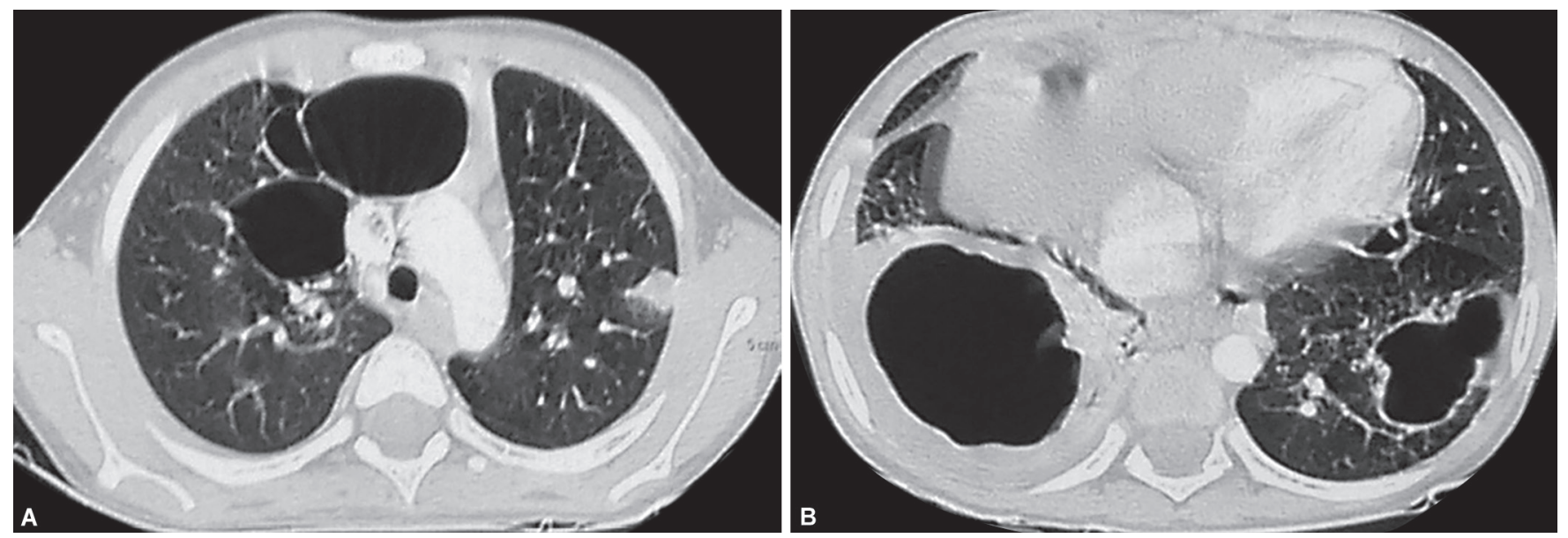

Figs 2A and B: CT chest: (A) Anterior extension of right pneumatoceles; (B) Pneumatoceles affecting bilateral lower lobes the left hemithorax for detection of pneumothorax. Intraoperative $\mathrm{SpO}_{2}$ ranged from 91 to $98 \%$ during one lung ventilation, which was managed by increasing $\mathrm{FiO}_{2}$ to $80 \%$. End tidal $\mathrm{CO}_{2}$ ranged from 35 to $45 \mathrm{~mm} \mathrm{Hg}$. Analgesia was maintained by intermittent intravenous fentanyl (total $6 \mu \mathrm{g} / \mathrm{kg}$ ), $400 \mathrm{mg}$ of paracetamol (infusion), and skin infiltration ( $10 \mathrm{~mL}$ of $0.125 \%$ bupivacaine). Intraoperatively, there was a sudden loss of tidal volume due to communication of right lower lobe pneumatocele with the lower lobe bronchus, which was managed by selective left lung ventilation at that time and surgical suturing. While excising the right upper lobe pneumatocele, the patient had few arrhythmias due to a close dissection near pericardium, which was managed conservatively. The chest wall was closed after insertion of the chest tube without air leak. The duration of the surgery was 4 hours with blood loss of $100 \mathrm{~mL}$. After reversal of residual neuromuscular blockade, the child was extubated. Post-extubation arterial blood gas analysis showed a pH of 7.34, $\mathrm{pO}_{2}$ of $167 \mathrm{~mm} \mathrm{Hg}, \mathrm{pCO}_{2}$ of $47 \mathrm{~mm} \mathrm{Hg}, \mathrm{HCO}_{3}^{-}$ of $25 \mathrm{mEq} \mathrm{L}^{-1}$, and $\mathrm{SaO}_{2}$ of $99.4 \%$.

In the high-dependency unit, in a propped-up position, oxygen (5 L/minute) was administered with Hudson's mask. Postoperative analgesia was maintained with IV tramadol $20 \mathrm{mg} 6$ hourly and IV paracetamol $300 \mathrm{mg} 8$ hourly. The patient recovered without any complications and was discharged on the eighth postoperative day.

\section{Discussion}

Hyperimmunoglobulin-E syndrome is a multisystem involvement of the immune system, skin and soft tissue, pulmonary, musculoskeletal, dental, cranial, and vascular systems. ${ }^{4,5,7}$ The National institutes of health $(\mathrm{NIH})$ clinical scoring system is an important evaluation system; scores $\geq 40$ suggest HIES. ${ }^{10}$ These patients may have repeated hospitalizations with multiple anesthesia exposures for various procedures such as drainage of abscess/empyema, bronchoscopies, lung resections, and scoliosis correction surgery. Perioperative assessment should focus on the severity of multisystem involvement.

Patients with HIES have distinct facial features such as prominent forehead with deep-set eyes, broad nasal bridge, a bulbous nose, and abnormal dentition.,11 Dermatological manifestations include recurrent staphylococcal abscesses, eczematous rash, and mucocutaneous candidiasis. Skeletal anomalies include osteopenia, hyperextensibility, degenerative 
joint disease, pathological fractures, discrepancy in limb length, and scoliosis. ${ }^{3,4,7}$ This demands careful positioning of patient under anesthesia.

Pulmonary involvement includes recurrent abscesses and pneumonia, pneumatocele, bronchiectasis, empyema, spontaneous pneumothorax, and bronchopleural fistula. Incidence of post-infectious pneumatocele formation can be up to $80 \%$ in these patients. ${ }^{12,13}$

Children presenting with recurrent pneumonias may have mild to severe respiratory distress. Preoperative assessment of respiratory function is very important to predict the postoperative recovery. This should include detailed history, clinical examination, chest X-ray, CT scan, arterial blood gases, and PFT to determine the cardiorespiratory reserve. PFTs are frequently abnormal because of a restrictive defect and difficult to perform in young children. The increased pressures in spirometry may increase the risk of rupture of pneumatocele. This child had pulmonary, cardiac, cutaneous, and dental involvement.

Indications for surgical intervention for pneumatocele are involvement of more than $50 \%$ of hemithorax with severe atelectasis, development of tension pneumatoceles leading to pneumothorax, a secondary infection of the pneumatocele, and cardiovascular compromise. Our patient had all these indications except cardiovascular compromise with recurrence of pneumatocele further complicated by immunodeficiency.

Induction of anesthesia is very challenging in cases of bilateral large pneumatoceles. Endotracheal intubation with IPPV in patients with tension pneumatoceles is a high-risk procedure because patients can develop pneumothorax progressing to hemodynamic instability. If unrecognized and untreated, this can result in respiratory failure and even cardiac arrest. Nitrous oxide should be avoided because of risk of pneumothorax following the rupture of pneumatoceles. In our case, the risk of contralateral pneumothorax was very high, hence vigilant monitoring of hemodynamic (IBP, $\mathrm{CVP})$ and respiratory parameters $\mathrm{SpO}_{2}, \mathrm{ETCO}_{2}$, airway pressures, stethoscope under contralateral hemithorax) were vital. Selective bronchial intubation and isolation allowed effective ventilation in our case.

Considering the immunodeficiency, perioperative prophylactic antibiotics, and strict asepsis for all cutaneous punctures (including venous access and regional anesthesia) is mandatory. Regional anesthesia is not contraindicated; however, the benefit of regional anesthesia should be considered against the risk of abscess formation in an immunocompromised patient. ${ }^{14}$ Formation of epidural abscess and late detection has been reported, ${ }^{15}$ hence we avoided epidural analgesia. These patients also exhibit various allergies. Hence, cross-sensitivity of propofol with egg or soya allergy must be kept in mind. ${ }^{6}$
To summarize, successful anesthesia management of such patients includes perioperative chest optimization, strict asepsis, prophylactic antibiotics, careful positioning, lung isolation, avoiding $\mathrm{N}_{2} \mathrm{O}$, a high index of suspicion for contralateral pneumothorax, vigilant monitoring with adequate systemic analgesia. Repeated surgeries and anesthesia exposure of these 'frequent fliers' should be kept in mind along with recurrence of pneumatoceles.

\section{References}

1. Davis SD, Schaller J, Wedgwood RJ. Job's Syndrome. Recurrent, "cold", staphylococcal abscesses. Lancet 1966;1:1013-1015. DOI: 10.1016/ S0140-6736(66)90119-X.

2. Grimbacher B, Holland SM, Gallin Jl, et al. Hyper-lgE syndrome with recurrent infections: an autosomal dominant multisystem disorder. $\mathrm{N}$ Engl J Med 1999;340:692-702. DOI: 10.1056/NEJM199903043400904.

3. Freeman AF, Holland SM. The hyper-IgE syndromes. Immunol Allergy Clin North Am 2008;28:227-291. DOI: 10.1016/j.iac.2008.01.005.

4. Grimbacher B, Holland SM, Puck JM. Hyper-IgE syndromes. Immunol Rev 2005;203:244-250. DOI: 10.1111/j.0105-2896.2005.00228.x.

5. Pietrucha B, Heropolitañska-Pliszka E, Cukrowska B, et al. Autosomal dominant hyper-lgE syndrome-presentation of symptomatology and review of the literature. Ann Diagn Paediatr Pathol 2008;12:45-48.

6. Wu J, Chen J, Tian ZQ, et al. Clinical manifestations and genetic analysis of 17 patients with autosomal dominant hyper-lgE syndrome in Mainland China: new reports and a literature review. J Clin Immunol 2017;37:166-179. DOI: 10.1007/s10875-017-0369-7.

7. Freeman AF, Holland SM. Clinical manifestations, etiology, and pathogenesis of the hyper-IgE syndromes. Pediatr Res 2009;65: 32R-37R. DOI: 10.1203/PDR.0b013e31819dc8c5.

8. Bosenberg AT. Anesthesia and Job syndrome. South Afr J Anaesth Analg 2008;14:11-14. DOI: 10.1080/22201173.2008.10872549.

9. Kulkarni P, Shah R, Priyanka VN. Anesthetic management in a child with Job's syndrome. Anesth Essays Res 2012;6:223-225. DOI: 10.4103/0259-1162.108342.

10. Woellner C, Gertz EM, Schaffer AA, et al. Mutations in STAT3 and diagnostic guidelines for hyper-IgE syndrome. J Allergy Clin Immunol 2010;125:424.e8-432.e8. DOI: 10.1016/j.jaci.2009.10.059.

11. Borges WG, Hensley T, Carey JC, et al. The face of Job. J Pediatr 1998;133:303-305. DOI: 10.1016/S0022-3476(98)70243-4.

12. Freeman $A F$, Kleiner DE, Nadiminti $\mathrm{H}$, et al. Causes of death in hyperIgE syndrome. J Allergy Clin Immunol 2007;119:1234-1240. DOI: 10.1016/j.jaci.2006.12.666.

13. Freeman AF, Renner ED, Henderson $C$, et al. Lung parenchyma surgery in autosomal dominant hyper-IgE syndrome. J Clin Immunol 2013;33:896-902. DOI: 10.1007/s10875-013-9890-5.

14. Tapper JB, Giesecke AH. Spinal Anesthesia in a child with Job's syndrome, pneumatoceles and empyema. Anesthesia 1900;45: 378-380. DOI: 10.1111/j.1365-2044.1990.tb14780.x.

15. Wong SS, Smith PR, Ayaz A. Hyperimmunoglobulin E syndrome with persistent vertebral osteomyelitis due to methicillin-resistant Staphylococcus aureus: case report and review of the literature. ID Cases 2014;1:12-13. DOI: 10.1016/j.idcr.2014.02.002. 